\title{
ARRIÈRÉS PÉDAGOGIQUES: NAISSANCE DE L'ÉLÈVE CAPACITAIRE DANS LES DERNIÈRES RECHERCHES DE BINET DANS LES ÉCOLES PARISIENNES EN 1908-1910
}

\author{
Bernard Andrieu \\ Université Paris-Descartes, EA 3625 TEC \\ Associé à l'UMR 6872 ADES CNRS et coord du GDRI 836 CNRS \\ bernard.andrieu@parisdescartes.fr
}

\begin{abstract}
RÉSUMÉ
L'élève capacitaire est autant celui qui pathologiquement manifesterait des carences et des déficits neurocognitifs que Binet et Simon décrivent comme un enfant anormal mais concerne aussi avec Vaney et les commissions pédagogique de la Société Binet-Simon les classes ordinaires dans lesquelles les différences de niveau d'intelligence ne sont pas réalisés. Epistémologiquement le projet de Binet avec d'une part le psychiatre Théodore Simon et d'autre part avec le directeur d'école Victor Vaney est double: - L'éducabilité peut être comprise dans une classification psychiatrique qui, depuis Belhomme jusqu'à Bourneville, propose une graduation dans les capacités d'apprentissage en délimitant les possibilités d'apprentissage, ce que nous appelons le niveau capacitaire de lélève dès la petit école de Borneville; - Alfred Binet travaillant avec Victor Vaney au laboratoire de l'école de la Grange aux Belles mais aussi dans les autres écoles parisiennes via les autorisations obtenues auprès de l'inspecteur Belot pour enquêter comme nous le montrons dans ces archives auprès des arriérés pédagogiques des classes ordinaires.
\end{abstract}

Mots-clés: Educabilité. Capacitaire. Pédagogie.

\section{ARRIÈRÉS PÉDAGOGIQUES: THE BIRTH OF THE CAPACITY STUDENT IN THE LATEST BINET RESEARCH IN THE PARISIAN SCHOOLS IN 1908-1910}

\begin{abstract}
The capacitive pupil is as much the pathologically deficient and neurocognitive deficits that Binet and Simon describe as an abnormal child but also concerns with Vaney and the pedagogical commissions of the Binet-Simon Society the ordinary classes in which differences in level Intelligence are not realized. Epistemologically the project of Binet with on the one hand the psychiatrist Theodore Simon and on the other hand with the school principal Victor Vaney is double: - Educability can be understood in a psychiatric classification which, from Belhomme to Bourneville, proposes a graduation in the capacities of learning by delimiting the possibilities of learning, what we call the capacity level of the student from the small school of Borneville; - Alfred Binet working with Victor Vaney in the laboratory of the Grange aux Belles school but also in the other Parisian schools via the authorizations obtained from Inspector Belot to investigate as we show in these archives to the educational arrears of the classes ordinary.
\end{abstract}

Keywords: Educability. Pedagogy. Capacitive pupil. 


\title{
ARRIÈRÉS PÉDAGOGIQUES: O NASCIMENTO DA CAPACIDADE NO ALUNO NA ÚLTIMA PESQUISA DE BINET NAS ESCOLAS PARISIENSES EM 1908-1910
}

\begin{abstract}
RESUMO
A capacidade do estudante como alguém que manifesta deficiências patológicas e déficits neurocognitivos que Binet e Simon descrevem como uma criança anormal, mas também no que diz respeito a Vaney e as placas educativas das classes regulares de Binet-Simon em que as diferenças no nível de inteligência não são realizadas. Epistemologicamente o projeto de Binet, por um lado, com o psiquiatra Theodore Simon e, por outro lado, com o Diretor da Escola Victor Vaney, é duplo: - Educabilidade pode ser entendida como uma classificação psiquiátrica, que, de Belhomme a Bourneville, oferece uma graduação na capacidade de aprendizagem na definição de oportunidades de aprendizagem, o que chamamos de nível de capacidade do aluno na pequena escola de Borneville; - Alfred Binet trabalhando com Victor Vaney no laboratório escola da Grangeaux Belles, mas também em outras escolas parisienses através das permissões obtidas do inspector Belot para investigar como mostram estes arquivos os atrasos educacionais das classes ordinárias.
\end{abstract}

Palavras-chave: Educabilidade. Capacidade. Pedagogia.

\section{ARRIÈRÉS PÉDAGOGIQUES: NACIMIENTO DEL ALUMNO CAPACITAIRE EN LAS ÚLTIMAS INVESTIGACIONES DE BINET EN LAS ESCUELAS PARISINAS EN 1908-1910}

\section{RESUMEN}

La capacidad del estudiante como uno que se manifiestan patológicamente carencias y déficits neurocognitivos que Binet y Simon describen como un niño anormal, sino también con respecto Vaney y las juntas de educación de las clases regulares de Binet-Simon compañía en las que las diferencias en el nivel la inteligencia no se realizan. Epistemológicamente redactar Binet con una mano psiquiatra Theodore Simon y también con el director Victor Vaney es doble: - La educabilidad puede estar en una clasificación psiquiátrica, que desde Belhomme a Bourneville, ofrece una graduación en la capacidad de aprendizaje en la definición de las oportunidades de aprendizaje, lo que llamamos el nivel de capacidad en la pequeña escuela lélève de Borneville; - Alfred Binet trabajar con Victor Vaney el laboratorio de la escuela en la Grange aux Belles sino también en otras escuelas de París a través de los permisos obtenidos del inspector de Belot para investigar como mostramos en este archivo con clases atrasos educativos ordinario

Palabras claves: Educabilidad, La capacidad, Education.

\section{LE CONTEXTE}

Dans le courant de 1906, Alfred Binet négocia avec le Directeur de l'Enseignement de la Seine la création immédiate de quelques classes de perfectionnement. Il souhaitait en effet à bon droit qu'un fonctionnement expérimental précédât toute initiative législative propre à les multiplier et à leur conférer un statut, car c'est évidemment à la vérification de l'éducabilité 
scolaire des débiles qu'était suspendue la pertinence d'une telle structure. En sollicitant le réseau de la Société libre pour l'étude psychologie de l'enfant, il multiplia démarches et instances, de sorte que la première "classe de perfectionnement" s'ouvrit en février 1907, la seconde en avril et la troisième en juin.

Epistémologiquement le projet de Binet avec d'une part le psychiatre Théodore Simon et d'autre part avec le directeur d'école Victor Vaney est double: l'éducabilité peut être comprise dans une classification psychiatrique qui, depuis Belhomme jusqu'à Bourneville, propose une graduation dans les capacités d'apprentissage en délimitant les possibilités d'apprentissage, ce que nous appelons le niveau capacitaire de l'élève dès la petit école de Borneville; Alfred Binet travaillant avec Victor Vaney au laboratoire de l'école de la Grange aux Belles mais aussi dans les autres école parisienne via les autorisations obtenues auprès de l'inspecteur Belot pour enquêter comme nous le montrons dans ces archives auprès des élèves de classes ordinaires:

Tableau 1. Classification.

\begin{tabular}{|c|c|c|c|}
\hline & $\begin{array}{l}\text { Belhomme } \\
1824 \\
\text { Bourneville } \\
\end{array}$ & $\begin{array}{c}\text { Binet } \\
\text { Simon }\end{array}$ & $\begin{array}{l}\text { Binet } \\
\text { Vaney }\end{array}$ \\
\hline Incurables & $\begin{array}{l}\text { idiots, } \\
\text { gâteux, } \\
\text { épileptiques } \\
\text { ou non mais } \\
\text { invalides et } \\
\text { réputés } \\
\text { incurables }\end{array}$ & $\begin{array}{l}\text { Démence } \\
\text { précoce } \\
\text { Binet/Simon } \\
1910\end{array}$ & \\
\hline Petite école & $\begin{array}{l}\text { idiots, } \\
\text { gâteux, } \\
\text { épileptiques } \\
\text { ou non mais } \\
\text { valides }\end{array}$ & $\begin{array}{l}\text { l'idiot aurait un } \\
\text { développement } \\
\text { de } 0 \text { à } 2 \text { ans }\end{array}$ & \\
\hline Grande école & $\begin{array}{l}\text { propres, } \\
\text { valides, } \\
\text { imbéciles, } \\
\text { arriérés et } \\
\text { épileptiques }\end{array}$ & $\begin{array}{l}\text { l'imbécile de } 2 \text { à } \\
7\end{array}$ & \\
\hline $\begin{array}{l}\text { Educabilité } \\
\text { capacitaire }\end{array}$ & $\begin{array}{l}\text { Indisciplinés } \\
\text { (Bourneville } \\
1897 \text { ) }\end{array}$ & $\begin{array}{l}\text { le débile de } 7 \text { à } \\
10 .\end{array}$ & $\begin{array}{l}\text { Les cancres (Retard } \\
\text { scolaire de } 3 \text { ans) Binet } \\
1907 \\
\text { Les enfants anormaux } \\
\text { Les élèves paresseux }\end{array}$ \\
\hline
\end{tabular}

Source: Elaboration de l'auteur.

L'élève capacitaire est autant celui qui pathologiquement manifesterait des carences et des déficits neurocognitifs que Binet et Simon décrivent comme un enfant anormal mais 
concerne aussi avec Vaney et les commissions pédagogique de la Société Binet-Simon les classes ordinaires dans lesquelles les différences de niveau d'intelligence ne sont pas réalisés. Dans sa commission des anormaux de la Société le 19 février 1904, la perfectibilité des anormaux par rapport au réfractaires est clairement posée:

\begin{abstract}
Voici les termes exacts de la proposition qui a été votée par la Commission des anormaux:

$1^{\circ}$ Que dans les écoles primaires, les enfants jugés réfractaires à l'éducation, à renseignement ou à la discipline de l'école, ne soient plus renvoyés sans avoir été soumis à un examen médico-psychologique;

$2^{\circ}$ Que ces enfants, s'ils sont reconnus comme anormaux perfectibles, soient groupés dans une classe spéciale annexée à l'école, ou dans un établissement spécial;

$3^{\circ}$ Qu'à titre de démonstration, une classe spéciale pour anormaux perfectibles soit ouverte dès à présent dans l'une des écoles de la Ville de Paris, notamment dans l'école de la rue Jenner, voisine de la Salpêtrière.
\end{abstract}

Binet précise lui-même, à la suite de ce rapport de la Commission signé Louette, déjà que la perception de cette perfectibilité doit être tangible et scientifique:

La Commission des anormaux s'est ensuite occupée, à ma demande, de réaliser un vaste plan de recherches, dont le succès a besoin du concours de tous. Il s'agit, en effet, d'arriver à établir scientifiquement quelles sont les différences corporelle et mentales qui séparent l'enfant normal et l'enfant anormal; il s'agit de préciser ces différences, de les mesurer en quelque sorte, de manière que leur perception cesse d'être une affaire de tact et d'intuition, pour devenir quelque chose d'objectif et de tangible. Si la Commission arrive à remplir ce programme, elle pourra se rendre cette justice d'avoir fait beaucoup pour le sort des anormaux. (BINET, 1904b, p. 506).

Binet était déjà particulirement lucide sur cette mise à l'écart des "arriérés pédagogiques" par la commission ministèrielle, alors même que l'enjeu est bien là pour la psychologie:

La Commission ministérielle d'études sur les enfants anormaux et arriérés s'est réunie deux fois, le $1^{\text {er }}$ décembre et le 15 décembre, au Ministère de l'Instruction publique, sous la présidence de M. Léon Bourgeois. Dans ces deux premières séances, d'importantes résolutions ont été prises. Il a été décidé que la Commission commencerait ses travaux par une statistique des enfants arriérés et anormaux qui existent actuellement en France. Cette statistique sera obtenue au moyen d'une enquête par questionnaires adressés notamment aux instituteurs. La confection de ce questionnaire a été confiée à une sous-commission, composée de MM. Baguer, Binet, Bourneville et Robin.

La Commission a décidé, en principe, que les seuls anormaux et arriérés de l'intelligence et des sentiments moraux seraient portés sur les tables de l'enquête; elle a par conséquent éliminé ces enfants qu'on appelle d'ordinaire et assez improprement des arriérés pédagogiques, et qui ne sont point des arriérés, mais tout simplement des ignorants par suite de fréquentation insuffisante de l'école. Ces enfants sont des normaux et doivent être maintenus dans les écoles ordinaires et normales. (BINET, 1904b, p. 346). 
C'est pourtant ces arriérés pédagogiques qui doivent décider de l'incommensurabilité entre la classification psychiatrique et la classification pédagogique. Le cancre, comme l'élève paresseux dont parle A. Binet dans les articles de la période que nous analysons plus bas, n'est donc pas un arriéré ce qui l'assimilerait à une catégorie psychiatrique. Pourtant Binet utilise le terme lorsque qu'il évalue la céphalométrie dans les écoles (BINET, 1901) sous l'influence de Simon qui a publié en 1900 un texte sans Binet sur les enfants arriérés de la colonie de Vaucluse sans parvenir à contenir ces arriérés dans la catégorie de ceux qui sont atteints de débilités intellectuelles ou morales et qui "peuvent ainsi présenter tous les degrés jusqu'à l'imbécilité ou même, par exception, l'idiotie" (SIMON, 1900, p. 431).

Dans son article qui expose des recherches menés dans les écoles entre décembre 1900 et janvier 1901 sur 50 élèves intelligents et 50 inintelligents, Binet justifie, à partir des renseignements recueillis auprès des maitres d'école, l'emploi du terme d'arriéré pour de "vrais anormaux" (BINET, 1901, p. 414): voulant mesurer la différence céphalométrique entre les élèves d'élite, les élèves moyens et les élèves arriérés, en ayant éliminés de ce groupe tous les élèves ayant eu des antécédents pathologique, développementaux ou de maladies ainsi que les nationalité étrangères ainsi que ceux d'origine israélite ou d'autres régions que Paris, Binet constate, avec un certain étonnement, que cette différence n'a pas d'explication sans une étude des conditions du "développement anormal" (BINET, 1901, p. 425) de ces élèves arriérés.

Dans l'enquête sur "la psychologie des cancres" (ANDRIEU, 2013) qui date des années 1908-1910 et que nous avons retrouvé et publié, Binet abandonne toute référence à un développement anormal de l'encéphale pour rechercher des causes sociales, morales et de travail pour justifier le classement de ces élèves qui ont 3 ans de retard sans être arriérés physiologiquement. L'arriération scolaire n'est pas la même que l'arriération mentale. Le cancre doit dont être défini dans un retard scolaire de 3 ans et sur 223 élèves étudiés ayant un retard de 3 ans, seulement 24 ont une déficience d'intelligence seule sans complication.

\section{LE BIAIS MÉTHODOLOGIQUE D'ALFRED BINET}

Dans ses travaux d'anthropologie scolaire Binet avait déjà pu mesurer le crâne des enfants pour démontrer, contre la phrénologie, qu'aucun indicateur physique ne parvenait à distinguer l'élève intelligent de l'élève idiot: ni la circonférence du crâne, ni le volume crânien, ni ses difformités développementales ne sont significativement différentes, la seule surface physique ne suffisant pas pour déterminer la qualité de l'activité cognitive. 
Dans son article de 1900 sur "Recherches sur la technique de la mensuration de la tête vivante" (BINET, 1900a), Binet définit l'inintelligence à partir de la désignation par les directeurs des élèves les plus intelligents et les moins intelligents de leur école " en tenant seulement compte de l'intelligence naturelle et non, de l'application au travail" (BINET, 1900a, p. 317); le travail de mensuration suppose que les interrogations de Binet sur les fiches qu'il établit porte "sur l'hérédité morale et médicale des enfants, leur état de santé, leur intelligence, leur caractère et leur activité physique" (BINET, 1900a, p. 318). Dans son article de 1900, "Recherches complémentaires de céphalométrie sur 100 enfants d'intelligence inégale choisis dans les écoles primaires du département de Seine-et-Marne” (BINET, 1900b), Binet distingue parmi les 100 enfants étudiés les intelligents, parmi lesquelles "des retardataires et des arriérés" (BINET, 1900b, 375), des “inintelligents" (BINET, 1900b, p. 375) sur la base d'une intelligence inégale.

Ainsi l'inintelligence est une norme négative dont l'inégalité est le principal critère de distinction entre les différents élèves. L'inégalité physique, grâce à l'anthropologie scolaire qu'établit Binet dans les écoles avec la mesure de la tension artérielle, de la force musculaire, de la taille du corps et du volume crânien, avait démontré comment l'intelligence et l'inintelligence ne relevaient pas d'une différence naturelle entre les sujets étudiés.

Dans son article "Recherches sur la technique de la mensuration de la tête vivante" (BINET, 1900c), Binet, tout en reconnaissant que “ c'est peut-être le point faible de ce travail” (BINET, 1900c, p. 317), dénonce lui-même un biais méthodologique qui n'aura pas été sans conséquence sur la question de la définition de l'inintelligence et sur la volonté du psychologue, comme nous le montrons dans ces archives, de définir lui-même ces critères: dans une recherche sur la relation entre la conformation de la tête et le degré d'intelligence des élèves, "une étude des extrêmes" (BINET, 1900c, p. 317) devait rendre " plus visible" la différence entre les intelligents et les inintelligents.

Or Binet ne s'appuie pas pour sélectionner sur sa propre méthode, il utilise une sélection faite par d'autres, en l'occurrence ceux qui ont déjà classé les élèves dans une des deux catégories en fonction de leur propre critère, comme si Binet devait confirmer ou invalider la discrimination entre les deux, ou se placer devant une telle situation pour finalement en révéler l'inefficacité méthodologique:

Pour ce choix des extrêmes, je m'en suis entièrement remis aux directeurs et professeurs des écoles. J'ai donc prié les directeurs de me désigner les sujets les plus intelligents et les moins intelligents de leur école, parmi les enfants âgés de 11 à 13 ans, sans distinction de classe, et en tenant seulement compte de l'intelligence naturelle et non de l'application au travail. (BINET, 1900c, p. 317). 
Ce biais méthodologique, dont Binet est conscient et qu'il n'aura de cesse de corriger jusque dans l'élaboration de l'échelle métrique, indique bien comme le système de sélection scolaire reposait sur le jugement arbitraire et l'expérience didactique des enseignants: l'intelligence naturelle, dont les critères scientifiques n'étaient pas établis, est repérée par la projection de l'habitude de la distinction scolaire entre les élèves d'élite et les autres. Cette distinction fabrique de l'handicapabilité des élèves avant même que Binet ne parvienne à préciser, à travers ses études sur les corpus, les critères “ l'hérédité morale et médicale des enfants, leurs état de santé, leur intelligence, leur caractère et leur activité physique” (BINET, 1900c, p. 318). Est-ce tant l'école qui handicape l'élève que des facteurs exogènes (familiaux, économiques et sociaux) dont l'influence doit être mesurée pour favoriser l'inclusion équitable d'enfants dit anormaux?

\section{LA QUESTION DU RENDEMENT SCOLAIRE}

La question du rendement scolaire se pose dans les savoirs prescrits à des élèves qui ne sont pas toujours en capacités et en âge de pouvoir les comprendre pour les réutiliser comme dans le calcul, la grammaire et l'orthographe. Mais aussi dans l'efficacité des méthodes d'évaluation du retard scolaire des enfants car cela peut les maintenir ou non en classe ordinaire. Cette nouvelle question est le moyen de réfléchir à la nature des gains en calcul et orthographes:

\footnotetext{
Ce gain suréquilibre sur les enseignements de tous ordres; mais il est un peu plus fort sur le calcul, qui était le plus en retard, un peu moins sur la lecture qui l'était le moins. C'est là, croyons-nous, une donnée intéressante. Et nous avons en même temps la légitime satisfaction de constater que c'est la première fois qu'on se préoccupe de ces questions de rendement, ou du moins qu'on cherche à remplacer une impression subjective par un calcul. Nous remarquerons que la méthode proposée ne peut être que provisoire: ce n'est qu'un essai, et il faudra le perfectionner. (BINET, 1908b, p. 152).
}

Binet en vient d'abord à interroger le contenu même des exercices donnés au élèves pour les évaluer. En privilégiant les sujets abstrait, seuls les plus agiles dans le traitement conceptuel comme il en avait établi le profil à propos de sa fille Marguerite comme nous l'avons montré dans la manuscrit de L'étude expérimentale de l'intelligence (ANDRIEU 2011). La naissance de ses filles, dont il pouvait quotidiennement et facilement observer l'évolution, allait diversifier son approche de l'intelligence, en les instaurant en objets de l' étude d'Alfred Binet, avant Piaget. Ainsi croit-il constater que "Marguerite" illustre le type observateur et "Armande" le type imaginatif. À la fin de l'étude expérimentale synthétisant 
les données recueillies, il établit la liste des critères qui l'ont conduit à les distinguer. Binet retrouve le même argument ici car il sait que l'éducation abstraite ne développe qu'une des aptitudes des enfants:

\begin{abstract}
Je me demande si en entraînant les élèves à des exercices de description, on leur donne également une avance pour des exercices de travail logique et de raisonnement, et si ceux qui arrivent ainsi à trousser joliment un tableau des choses vues sont devenus par là même plus habiles à traiter un sujet abstrait. Je me demande encore si, lorsqu'on développe beaucoup une des aptitudes des enfants, il n'en résulte point un petit déchet dans une autre branche de l'enseignement, si ce déchet est grand ou petit par rapport au gain obtenu d'autre part, ou si au contraire l'acquisition d'une aptitude spéciale n'entraîne pas comme une amélioration, une mise en forme de "l'intelligence générale. (BINET, 1910c, p. 166).
\end{abstract}

L'évaluation de l'anormalité des enfants en fonction du retard scolaire dans une comparaison d'une année:

On fait faire aux anormaux, à leur entrée dans la classe, une composition dictée d'orthographe, et une autre de calcul; cette dernière se compose d'une série graduée de problèmes. Les copies sont ramassées, on les corrige, mais on ne les montre point aux enfants. Puis on fait lire les enfants, et on apprécie exactement leur degré de lecture. Un an après, ou six mois après, on recommence les mêmes épreuves, exactement dans les mêmes conditions, et avec les mêmes textes. Les enfants ne peuvent pas s'en souvenir, à cause du temps écoulé et du nombre considérable d'exercices différents qui ont été faits depuis. En calculant les moyennes du retard, pour les trois exercices (orthographe, calcul, lecture) et pour tous ces enfants, on obtient les résultats suivants, pour la classe de filles, la seule que nous étudierons en détail:

Classe de filles. Moyenne du retard en avril 1907: 2 ans 54 148).

en mars 1908: 1 an 25. (BINET, 1908b, p.

Cet écart pose le problème de la perfectibilité de l'élève dit anormal au regard de la norme de normalité acceptatble dans la classe ordinaire. Ces chiffres ont besoin d'explication. Nous disons que le retard moyen, en calcul, orthographe et lecture, était, à l'entrée, de deux ans et demi; il n'était pas de trois ans, parce que nous avions admis quelques instables dont le retard était moindre, et aussi parce que nous avions fait quelques erreurs en mesurant le retard. Supposons maintenant que les enfants fussent restés dans leur école d'origine; comme ils ne progressent que d'une demi année en un an, leur retard se serait encore aggravé; au bout d'un an, il aurait été de deux ans et demi, + une demie, = trois ans. Et s'ils ne travaillaient pas du tout, s'ils étaient stationnaires, ils auraient, au bout d'un an, trois ans et demi de retard. Or, puisqu'ils ont gagné de ne plus être en retard que de un an 25, on leur a fait faire en un an le travail de deux ans et quart (BINET, 1908b, p. 149).

\title{
COMMENT LES INSTITUTEURS JUGENT-ILS L'INTELLIGENCE DE L'ÉCOLIER?
}

Alfred Binet est très sceptique sur la manière dont les instituteurs évaluent le niveau d'intelligence de leurs élèves: d'une part parce qu'il veut s'imposer avec son échelle métrique comme LE spécialiste de l'évaluation de l'intelligence mais aussi parce que la perception 
subjective des maîtres doit pouvoir être établie, afin d'étalonner ce que serait une mesure plus objective:

\begin{abstract}
Nous venons de faire connaître les résultats de la petite enquête par questionnaire que M. l'Inspecteur Belot a bien voulu faire parmi son personnel. Il y avait trois questions posées: $1^{\circ}$ comment vous rendez-vous compte habituellement du degré d'intelligence des enfants? Citez des exemples précis; $2^{\circ}$ Existe-t-il des cas où vous avez eu de la difficulté à vous rendre compte de l'intelligence d'un enfant, et où vous avez commis une erreur? $3^{\circ}$ Dans quelles circonstances ces difficultés et erreurs se sont-elles produites le plus souvent? (BINET, 1910d, p. 170).
\end{abstract}

Alfred Binet essaie de quantifier ces erreurs de jugements sur les déclarations des maîtres d'école car c'est moins les faits qu'il relève que les “appréciations de l'intelligence de l'enfant", révélant ainsi la part du jugment subjectif, malgré l'expérience professionnelle des instituteurs et institutrices. Remarquons entre parentèse, je l'ai souligné, le commentaire de Binet à la réponse de l'institutrice:

\begin{abstract}
Comme proportion, j'estime que sur 75 élèves environ, je ne rencontre pas habituellement plus de 3 enfants dont j'ai de la peine à apprécier l'intelligence. (Donc la proportion est de quatre pour cent. La correspondante cite comme exemple d'erreur le cas d'une jeune fille qu'elle jugeait peu intelligente mais elle fit la connaissance des parents, vit la jeune fille dans sa famille, et constata qu'elle avait une excellente intelligence des choses de la vie. Elle conclut qu' "être intelligent ne signifie pas seulement avoir de l'aptitude pour apprendre". - Cette dernière remarque est bien vraie; mais il y a beaucoup de professeurs qui ne la font pas !)? (BINET, 1910, p. 172).
\end{abstract}

Binet n'établit une analyse statistique qu'en extrayant une fréquence par un passage de la déclaration qualitative à un chiffre quantitatif. Ainsi

Un calcul, auquel il ne faut pas attacher trop d'importance, nous donne la série suivante: nous avons rapporté les résultats à 100 , et les chiffres suivant sont des pourcentages d'erreurs. 0\%: 3 fois; $1 / 2 \%$ : 1 fois; $2 \%$ : 1 fois; $3 \%$ : 1 fois; $5 \%$ : 4 fois; 10\%: 5 fois; 20\%: 1 fois; 25\%: 3 fois; 30\%: 2 fois. Valeur médiane: $7,5 \%$ Il résulterait de cette série, que la valeur médiane est de 7,5\%; ce qui signifie qu'on doit faire une erreur sur 13 ou 14 enfants. (BINET, 1910d, p. 179).

Alfred Binet dresse en conclusion des limites à l'appréciation subjectives des capacités d'intelligence des élèves par les instituteurs et institutrices (c'est nous qui soulignons):

\footnotetext{
Après avoir bien réfléchi, et nous être rendu compte que nous faisons ici surtout de l'interprétation subjective, nous proposons les conclusions suivantes:

$1^{\circ}$ L'appréciation de l'intelligence d'un élève est toujours une opération délicate; car ceux-là même qui se décernent l'infaillibilité reconnaissent que cette appréciation exige une longue observation, de un ou plusieurs mois, et qu'elle reste exposée à des causes d'erreurs dont ne peut pas toujours se débarrasser.

$2^{\circ}$ Les erreurs commises et reconnues par les Maîtres seraient, autant qu'on peut se fier à une moyenne, qui n'est là que pour fixer les idées, d'environ 1 sur 13.
} 
$3^{\circ}$ Je suppose qu'en général un peu d'amour-propre a dû se mêler à ces appréciations, et que leur exactitude a été plutôt surestimée. Pour être instituteur, on en est pas moins homme. (BINET, 1910d, p. 182).

Mais plus radical est le jugement que Binet porte sur le défaut d'évaluation de l'avenir professionnel de l'élève par l'aveuglement sur la vocation et la direction professionnelle à donner aux élèves:

Il y a lieu aussi de remarquer que dans beaucoup de cas, lorsque le Maître s'est trompé, il n'a pas dû s'en apercevoir. Les démentis qu'on nous cite comme les plus frappants ont été fournis par la vie ultérieure de l'élève. Quand on ne l'a pas suivi, on n'a pas dû s'apercevoir qu'on s'était trompé. Une institutrice trouve dans un magasin de nouveautés une de ses anciennes élèves qui était, dit-elle, d'une passivité déconcertante; et elle est surprise de constater que cette élève est devenue une charmante vendeuse, pleine de grâce et de spontanéité. Mais se serait-elle aperçue de la métamorphose, si elle n'était pas entrée par hasard dans ce magasin de nouveautés? (BINET, 1910d, p. 182).

La critique est d'autant plus sévère que Binet veut établir une claire distinction scientifique entre l'appréciation de l'inteligence par les instituteurs/trices d'école et l'évaluation scientifique. Le critère de l'erreur d'appréciation serait le moyen d'établir cette distinction:

De plus, ces appréciations sur l'intelligence sont d'ordinaires faites dans des termes assez vagues: on dit d'un écolier qu'il est très intelligent, ou peu, ou dans une proportion moyenne. De tels jugements sont difficiles à contredire; on ne peut mettre à l'épreuve que des jugements précis. Ceux qui restent vagues échappent aux chances d'erreurs, mais ils perdent aussi les chances d'exactitude. Toutes ces raisons me font admettre que la proportion d'erreur doit être plus grande que 1 sur 10. En résumé, j'espère ne pas forcer dans un sens quelconque la conclusion de cette enquête en disant que, d'après l'opinion des instituteurs, apprécier l'intelligence d'enfant est une affaire toujours délicate, et que les chances d'erreurs ne sont pas négligeables. (BINET, 1910, p. 182).

\section{LES QUEUES DE CLASSE ET LA PSYCHOLOGIE DE LA PARESSE}

Les arriérés pédagogiques trouvent dans les conditions sociales du travail des élèves des raisons que la psychologie des cancres avaient déjà qualifié de causes externes. Mais la paresse dans ce manuscrit était comprise à la fois comme une des causes personnelles (en 29 items: intelligence(24), paresse(1), audition(3), vue(1)) et une des causes mixtes qui regroupent plus de 84 items mais en mêlant les causes extrinsèques avec les causes personnelles: (Maladie et inintelligence: 11; Chétivité et intelligence: 5; Maladie et paresse; Mauvais enseignement et inintelligence: 2; Mauvaise vue et scol. irrég.: 1; Faute des parents et inintelligence: 1; Irrégularité et inintelligence: 32 ; Irrégularité et mauvais ens.: 1; Faute des parents et maladie; Maladie, inint., mauvais enseignement: 1; Intelligence, application, 
maladies: 1; Malade et irrégulier: 5; Maladie, paresse, scol. Irrég.: 3; Maladie, inint., irrég.: 8; Vision et maladie, irrég.: 4; Inintellig. et étranger: 1; Irrég. et paresse: 1).

Mais si l'irrégularité scolaire est l'item dont la fréquence revient en rapport mixte avec l'intelligence, avec la paresse, avec la maladie, Binet en conçoit un facteur d'aggravation à la santé scolaire: ainsi il convient de faire une différence entre la santé personnelle et la santé scolaire car si la première enferme l'élève dans son étiologie strictement physiologique, sinon héréditaire, comme la maladie, la mauvaise vue, l'anémie ou des maladies liées au mode d'existence des corps comme la chétivité, la tuberculose, la grippe, la coqueluche, les végétations ou la congestion cérébrale. La santé scolaire doit prendre en compte l'accumulation des causes et leur mélange: ainsi mêler maladie-paresse- irrégularité scolaire vient immédiatement aggraver le cas de ces trois élèves, si bien que le traitement ne peut être seulement médical obligeant un diagnostic interdisciplinaire à partir du dossier singulier de chaque cas.

Pourtant (CHAPUIS, 2004) à l'enquête auprès de 103 élèves sur la paresse par Camille Boquillon dans le cadre de la commission des qualités et des défauts de l'enfant Alfred Binet reprend les conclusions de son enquête sur la psychologie des cancres mais en mettant l'accent sur ce qui serait les "vrais paresseux":

\begin{abstract}
Remarquons bien que l'appréciation de la quantité de paresseux a été faite par M. Bocquillon avec toutes sortes de précautions; ce n'est pas une impression subjective, arbitraire, dépendant à la fois de l'expérience qu'on a acquise et de la définition qu'on se donne de la paresse. Il a été procédé au contraire avec beaucoup de méthode; on a pris les enfants occupant le dernier cinquième de la classe, par conséquent ceux dont la paresse, si elle existe, produit des insuccès scolaires graves; et ensuite, on a cherché combien de ces "queues de classe" ont une excuse dans la chétivité du corps, les altérations des sens, ou la faiblesse de l'intelligence, ou autres causes analogues; et après avoir fait l'élimination de tous les excusés, on voit combien il reste peu de vrais paresseux; 2 pour cent, c'est une proportion infiniment petite. (BINET, 1909c, p. 155).
\end{abstract}

Dans cette discussion Alfred Binet remplace le terme de cause personnelle et causes mixtes qu'il avait définit dans la psychologie des cancres par le terme types purs et types mixtes:

\footnotetext{
Mais en revanche, il faut bien admettre que la paresse, comme cause accessoire, doit agir chez beaucoup d'élèves, soit d'une manière temporaire, soit d'une manière permanente, et ajouter son effet au concert des autres causes, tant physiques que morales, qui produisent un ralentissement du travail scolaire. Les types purs de paresse sont tellement rares qu'on peut se demander s'ils ne sont pas problématiques. Les types mixtes sont ceux qu'on rencontre le plus souvent, et ils ne sont certainement pas en quantité négligeable. (BINET, 1909c, p. 156).
} 
Plus fondamentalement la paresse ne peut, sur l'échelle de l'intelligence, être comprise comme un "abaissement de l'intelligence" car la paresse est une autre catégorie de comportement scolaire qui relèverait d'une "lésion psychologique":

\begin{abstract}
L'auteur du rapport paraît avoir hésité un peu sur la définition de la paresse. Cette définition-là ne ressort pas de son rapport, si ce n'est sous une forme négative; est paresse, tout insuccès scolaire qui ne s'explique ni par la chétivité, ni par l'abaissement de l'intelligence, ni par d'autres causes équivalentes. Cette forme négative de la définition suffisait d'ailleurs à son travail. L'enquête n'en comportait pas d'autre. Il aurait fallu entreprendre des recherches d'un genre bien différent pour essayer une définition positive, mettant le doigt sur la lésion psychologique qui constitue l'enfant paresseux. (BINET, 1909c, p. 157).
\end{abstract}

\title{
UNE ÉTUDE COGNITIVE GENRÉE SUR LA MÉMOIRE DES MOTS
}

Cette "lésion psychologique" que recherche Binet pour la paresse, nous la retrouvant dans ces deux feuilles d'archives retrouvées. Nous presentons ici ces archives inédites sur la mémoire des mots, difficilement datable sans doute entre 1907 et 1910 au moment où Alfred Binet mène des enquêtes dans les écoles parisiennes. Ces archives faisaient partie d'un fameux dossier vert trouvés dans l'appartement des petits filles Binet que j'ai pu recueillir et établir avant de les confier à la Bibliothèque Nationale de France avec l'aide de Mme Laurence Le Bras, conservatrice à la BNF et nos collègues Jacqueline Descarpentries, Alix Garnier \& Severine Parayre, afin de préciser le cadre juridique du don de la Famille Binet et obtenir une protection du fonds par une mise en disponibilité au public de chercheurs et au grand public. Chacun peut désormais travailler sur ces archives ${ }^{1}$. La perspective du Centenaire Binet, dont nous avions pu obtenir l'inscription du Centenaire ${ }^{2}$ dans les Célébrations Nationales en 2011, fut ainsi l'occasion d'établir deux textes ${ }^{3}$ et de les protéger par des publications de références et de réaliser grâce au soutien de l'Université de Lorraine à Alexandre Klein, un WeBinet ${ }^{4}$ dans la suite du colloque du Centenaire http://alfredbinet.univlorraine.fr.

En effet à travers le thème de la mémoire des mots, les différences garçons filles sont d'ordre psychologique et cognitif en fonction de l'âge. La présentation visuelle et celle auditive, mise en oeuvre par Belot comme par Binet, est une comparaison entre deux modes sensori-cognitif:

\footnotetext{
${ }^{1}$ archivesetmanuscrits.bnf.fr (cote: NAF 28884).

${ }^{2} \mathrm{https}: / /$ rechercheseducations.revues.org/749

${ }^{3}$ Ed (2011) Le journal de Madeline Binet, Archives Binet/ Célébrations Natonales. Ed., (2011) Les notes sur l'étude expérimentale de l'intelligence d'Alfred Binet, Archives Binet/ Célébrations Nationales.

${ }^{4} \mathrm{http}$ ://binet.hypotheses.org/190
} 
Or, on n'est jamais certain qu'une série de mots est exactement aussi difficile qu'une autre série, au point de vue de la mémoire de l'orthographe; il faudrait cependant que la difficulté fût égale, pour qu'on pût juger les procédés. M. Belot a paré à cette objection en faisant des combinaisons diverses avec les procédés et les séries des mots; ainsi, il a comparé les effets de la présentation visuelle et de la présentation auditive pour les mêmes séries de mots, avec des élèves différents; et le nombre considérable des élèves ayant servi de sujets a effacé toutes les différences individuelles tenant à leur diversité d'intelligence et de culture. (BINET, 1908, p. 105).

L'accent est mis sur la "diversité d'intelligence et de culture" qui pourra être établie par les réponses entre les deux modes visuels et auditifs: selon la constitution visuelle ou auditive de la mémoire et l'adaptation orthographique des série de mots doit se réaliser en fonction de l'âge comme on le remarque dans l'archive 1 avec les différents âges des filles. Alfred Binet vient critiquer chez la collaboratrice suisse Melle Metral de la Société ce qui serait une absence de distinction d'âge mental, qui est au fondement même du test élaboré par Alfred Binet et Théodore Simon:

Si je comprends bien le travail de Mlle Métral, elle n'a pas vu cette cause d'erreur, ou du moins elle n'en parle pas; elle s'est contentée d'opérer sur les 22 garçons de sa classe, qui tous ont appris l'orthographe des séries de mots tour à tour avec des procédés différents; de sorte que les résultats inégaux qu'elle enregistre peuvent tenir soit à la valeur différente des procédés, soit à la difficulté orthographique différente des séries de mots. Peu importe du reste que l'erreur que je signale ait produit ou non des conséquences graves dans le cas actuel. Il m'a paru surtout utile de rappeler à nos collaborateurs de l'étranger qu'il y a certaines règles d'expérimentation dont on ne doit pas se départir. (BINET, 1908, p. 108).

Dans l'Archive 1, 4 séries d'exercices de Mémoire de 100 mots sont proposées au garçons comme aux filles d'un même âge même si l'âge de 11 n'appartient qu'à la série des garçons: mots rappelés, mots reconnus, erreur de rappel, erreur de reconnaissance. La courbe progresse avec l'âge pour les mots rappelés tant chez les filles que chez les garçons avec une pointe à 11 ans pour les filles. Mais le nombre de mots retenus par la fille de 11 ans est seulement de 15 alors que le garçon de 12 ans en retient 19. 
Archive 1: Comparaison d'une école de garçons et d'une école de filles sur la mémoire des mots.

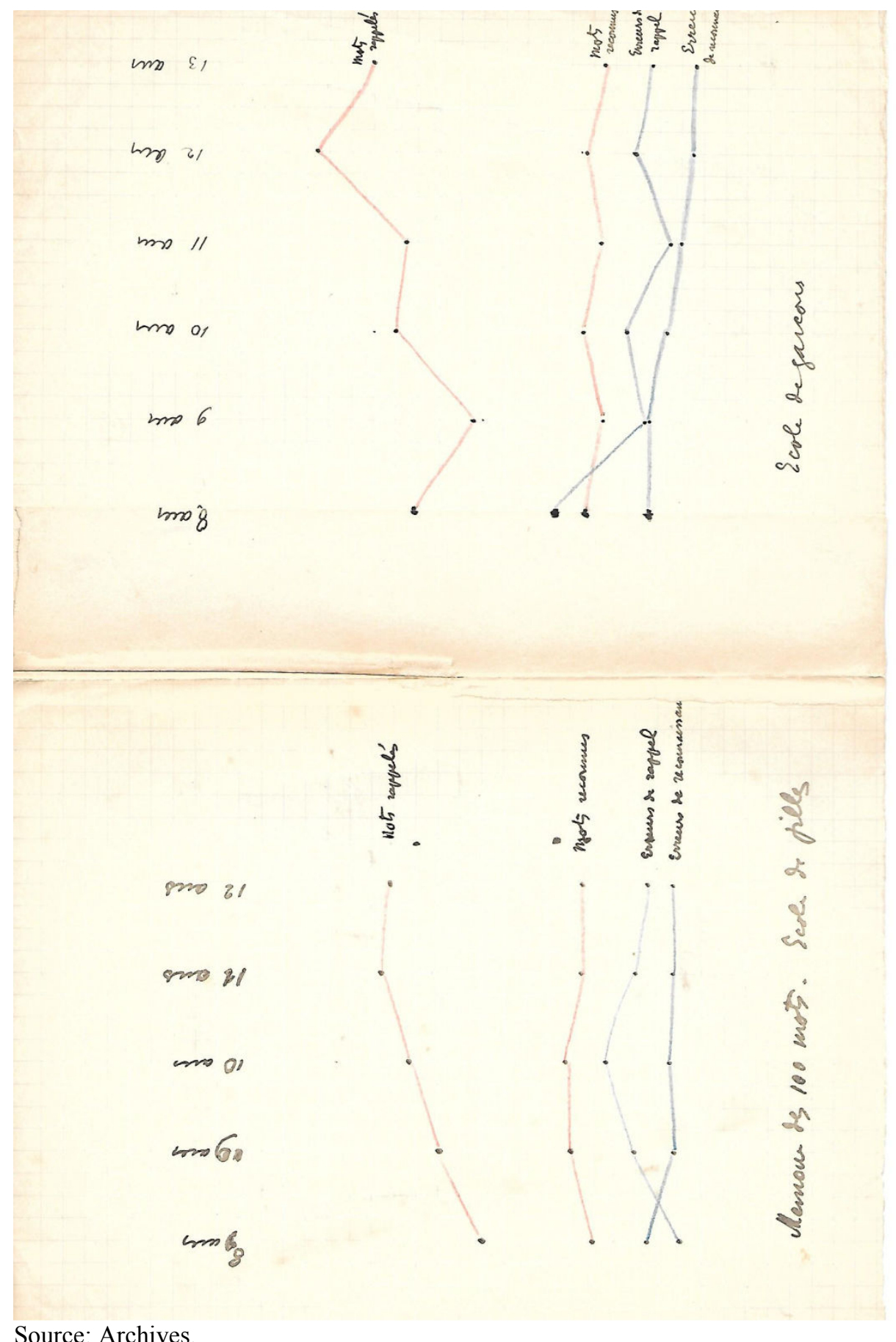

Source: Archives.

A l'inverse la fille de 8 ans retient 11 mots tandis que celui de 9 ans arrive à ce score seulement à cet âge. Il est difficile d'extraire des lois sur la mémoire à partir d'une comparaison dont nous ne savons s'il s'agit de résultat individuel, de groupe d'âge par sexe ou encore de comparaison simultanée. Mais il est significatif que l'écart entre mots rappelées et mots reconnus est moins grands chez les filles, plus cultivées à l'évidence que chez les garçons qui s'ils ont une mémoire quantitative plus grande ne parviennent pas à les 
comprendre. Le niveau d'erreur de reconnaissance est plus bas chez les filles entre 2 et 3 tandis que chez les garçons sont entre 4 et 5 .

\section{CONCLUSION}

Avec l'arrieré pédagogique, Binet, comme avec le cancre, parvient à dégager un nouvel objet épistémologique: celui de l'éducabilité perfectible d'un élève ni paresseux ni idiot. Il aura fallu déconstruire la naturalisation de l'intelligence par le cerveau mais aussi les erreurs d'appréciation de l'intelligence des élèves par les instituteurs pour dégager les ressources capacitaires de tout élève.

\section{RÉFÉRENCES}

ANDRIEU, B. Alfred Binet, Les notes sur l'étude expérimentale de l'intelligence. Archives Binet/ Célébrations Nationales, 2011 a.

. Genèse des types mentaux: des notes des tests familiaux d'Alfred Binet. L'étude expérimentale de l'intelligence, L’Année Psychologique, $\mathrm{n}^{\circ} 111,3$, Note Historique / Centenaire de la mort de Binet, p. 509-532, 2011 b.

. Madeleine ou Marguerite, quel sujet psychologique chez Alfred Binet? Bulletin de psychologie, Alfred Binet, cent ans après, Tome 64 (3), $\mathrm{n}^{\circ}$ 513, p. 213-218, $2011 \mathrm{c}$.

. Psychologie des cancres. Une archive inédite d'Alfred Binet. Recherches \& Educations, $\mathrm{n}^{\circ}$ 8, juin, p. 165-180, 2013.

BINET, A. Recherches sur la technique de la mensuration de la tête vivante. A.P., 7, p. 314$368,1900 a$.

. Recherches complémentaires de céphalométrie sur 100 enfants d'intelligence inégale

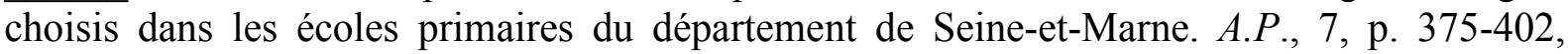
$1900 \mathrm{~b}$.

. Recherches sur la technique de la mensuration de la tête vivante. A.P., 7, p. 317, 1900.

Recherches de céphalométrie sur des enfants d'élite et arriérés des écoles primaires de Paris (déc.1900 et janvier 1901). A.P.,7, p. 412-429, 1901 a.

Avis. La Commission ministérielle pour les anormaux. Bul., 18, p. 506, 1904.

. A propos de récentes expériences de psychologie sur la mémoire de l'orthographe. Bul., 46, avril, p. 104-108, 1908a. 
. Les méthodes permettant de contrôler le rendement scolaire d'un enseignement, d'une classe ou d'une Ecole Application d'Enfants anormaux. Bul. 48, juin, p. 143-152, 1908 b. 1909a.

Ce que vaut l'Ecole primaire comme préparation à la vie. Bul., 52, févr., p. 58-63, 1909b.

L'école primaire, comme préparation à la vie. Bull., 54 avril mai 1909, p 101-108,

. A propos de l'enquête sur les enfants paresseux. Bul., 56, juill., p. 154-157, 1909c.

. Les signes physiques de l'intelligence chez les enfants. A.P.,16, p.1-30, 1910a.

. Correspondance; Mesure du niveau intellectual. Bul., 60, Févr., p. 89-92, 1910 b.

. Deux mots au sujet de la Communication de M. Bocquet. Bull., 63, p. 166, 1910c.

$1910 \mathrm{~d}$.

. Comment les instituteurs jugent-ils l'intelligence de l'écolier? Bul., 64, p. 172-82,

. Nouvelles recherches sur la mesure du niveau intellectuel chez les enfants d'école. $\overline{A . P ., ~ 17, p . ~ 145-201, ~} 1911$ a.

. Etude d'un procédé nouveau pour la mesure du niveau intellectual. Bul., 69, mars, p. 156-169, $1911 b$.

; SIMON, T. La mesure de l'intelligence chez les enfants (avec démonstrations). Bull. de la soc. clinique de médecine mentale, 9, p. 1-10, 1909. 70-71, p. 187-256, 1911.

. La mesure du développement de l'intelligence chez les jeunes enfants. Bul.

; VANEY, V. La mesure du degré d'instruction d'après des recherches nouvelles. Bul., 66, p. 1-14, 1910.

BOCQUILLON, C. Enquête sur la paresse, Bull, n 55, p. 120-144 et n 56, 1909.

CHAPUIS, É. Les enquêtes de psychologie de l'enfant, une industrie. Mil neuf cent. Revue d'histoire intellectuelle, $\mathrm{n}^{\circ} 22$, p. 77-94, 1/2004.

SIMON, T. Recherches céphalométriques sur les enfants arriérés de la colonie de Vaucluse. A.P., 6, 430-489, 1900. 